\title{
Bilinear Pseudodifferential Operators and the Hörmander Classes
}

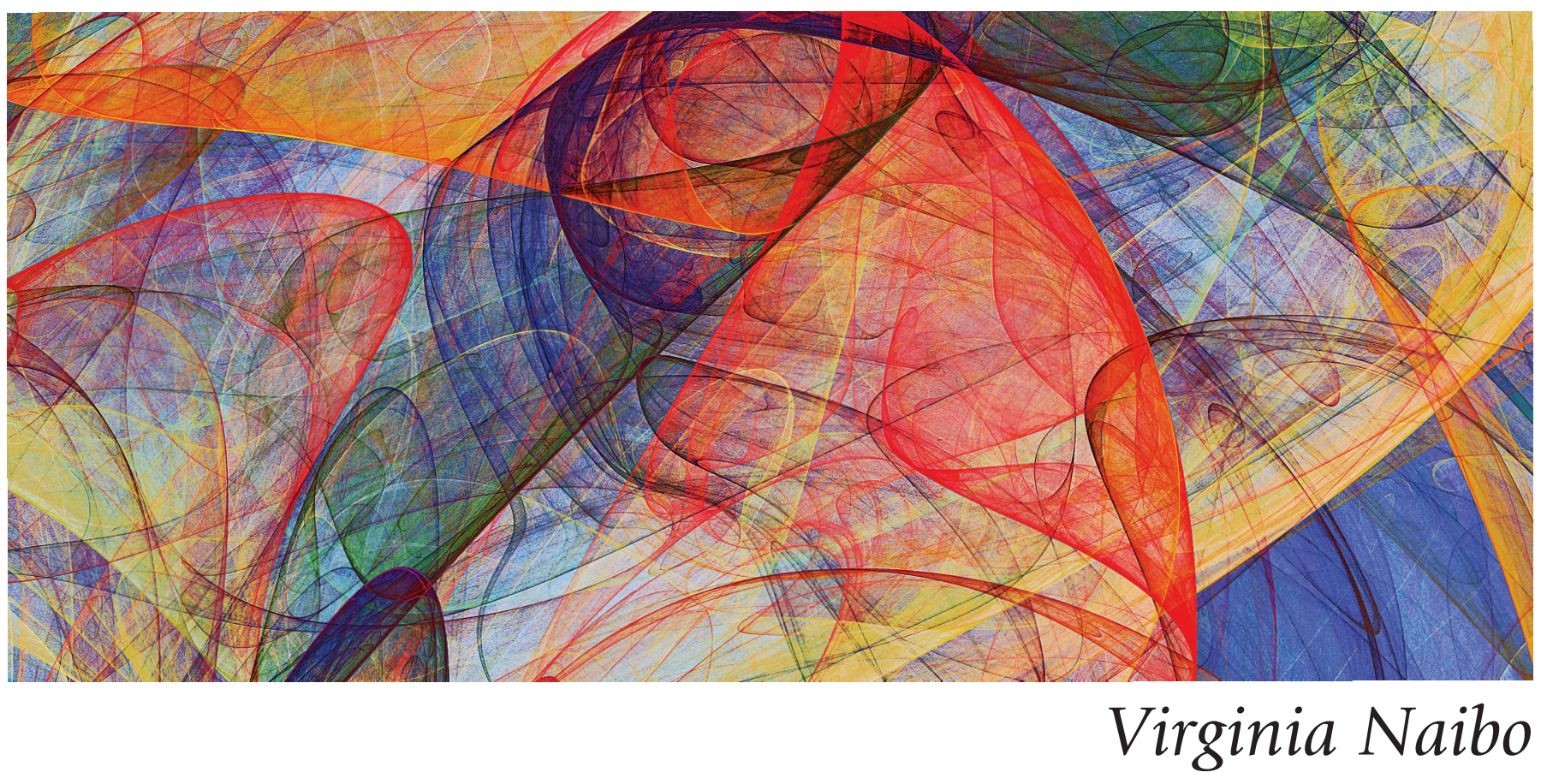

\section{Introduction}

As suggested by their name, bilinear pseudodifferential operators go beyond the class of differential operators. Their study is motivated by applications to analysis and partial differential equations as well as by the corresponding linear theory. This article is devoted to bilinear pseudodifferential operators associated to the so-called Hörmander classes. Different aspects of the theory will be described, with an emphasis on their boundedness properties in a variety of function spaces.

In order to motivate the definition of a bilinear pseudodifferential operator, consider

$$
L(f, g)(x)=\sum_{|\mu| \leq m_{1},|v| \leq m_{2}} a_{\mu, \nu}(x) \partial^{\mu} f(x) \partial^{\nu} g(x),
$$

Virginia Naibo is a professor of mathematics at Kansas State University. Her email address is vnaibo@ksu. edu.

Communicated by Notices Associate Editor Daniela De Silva.

For permission to reprint this article, please contact:

reprint-permission@ams.org.

DOI: https://doi.org/10.1090/noti2313 where $m_{1}, m_{2} \in \mathbb{N}_{0}, \mu, \nu \in \mathbb{N}_{0}^{n}$ are multi-indices, $a_{\mu, \nu}, f$, and $g$ are functions defined on $\mathbb{R}^{n},|\mu|=\mu_{1}+\cdots+\mu_{n}$ for $\mu=\left(\mu_{1}, \ldots, \mu_{n}\right), \partial^{\mu}=\partial_{1}^{\mu_{1}} \ldots \partial_{n}^{\mu_{n}}$, and similarly for $|\nu|$ and $\partial^{\nu}$. Recalling that the Fourier transform is defined for an integrable function $h$ on $\mathbb{R}^{n}$ as $\widehat{h}(\xi)=\int_{\mathbb{R}^{n}} h(x) e^{-2 \pi i x \cdot \xi} d x$ for $\xi \in \mathbb{R}^{n}$, basic properties give that the partial differential operator $L$ can be written as

$$
L(f, g)(x)=\int_{\mathbb{R}^{2 n}} P(x, \xi, \eta) \widehat{f}(\xi) \hat{g}(\eta) e^{2 \pi i x \cdot(\xi+\eta)} d \xi d \eta,
$$

where $x \cdot(\xi+\eta)$ stands for the dot product of $x$ with $\xi+\eta$ and $P(x, \xi, \eta)$ is the characteristic polynomial of $L$, that is,

$$
P(x, \xi, \eta)=\sum_{|\mu| \leq m_{1},|\nu| \leq m_{2}} a_{\mu, \nu}(x)(2 \pi i \xi)^{\mu}(2 \pi i \eta)^{\nu},
$$

with $\xi^{\mu}=\xi_{1}^{\mu_{1}} \cdots \xi_{n}^{\mu_{n}}$ for $\xi=\left(\xi_{1}, \ldots, \xi_{n}\right)$ and similarly for $\eta^{\nu}$.

We also note that if the functions $a_{\mu, \nu}$ are infinitely differentiable with bounded derivatives of all orders, then the characteristic polynomial $P$ satisfies the estimates

$$
\left|\partial_{x}^{\alpha} \partial_{\xi}^{\beta} \partial_{\eta}^{\gamma} P(x, \xi, \eta)\right| \leq C_{\alpha \beta \gamma}(1+|\xi|+|\eta|)^{m-(|\beta|+|\gamma|)}
$$


for all $x, \xi, \eta \in \mathbb{R}^{n}$, for all multi-indices $\alpha, \beta, \gamma \in \mathbb{N}_{0}^{n}$, for some constants $C_{\alpha \beta \gamma}>0$, and with $m=m_{1}+m_{2}$. Thus, when taking a derivative of $P$ with respect to a component of the frequency variables $\xi$ or $\eta$, the power on the right-hand side of (2) decreases by one; on the other hand, when taking a derivative of $P$ with respect to a component of the space variable $x$, the power on the right-hand side of (2) stays unchanged.

The expression on the right-hand side of (1) is an example of a bilinear pseudodifferential operator with symbol $P(x, \xi, \eta)$. More generally, a bilinear pseudodifferential operator is of the form

$$
T_{\sigma}(f, g)(x)=\int_{\mathbb{R}^{2 n}} \sigma(x, \xi, \eta) \widehat{f}(\xi) \hat{g}(\eta) e^{2 \pi i x \cdot(\xi+\eta)} d \xi d \eta,
$$

where $\sigma(x, \xi, \eta)$ is a complex-valued function defined for $x, \xi, \eta \in \mathbb{R}^{n}$ called the symbol of the operator. If $\sigma$ depends only on the frequency variables $\xi$ and $\eta, T_{\sigma}$ is usually referred to as a bilinear multiplier operator and $\sigma$ as a bilinear multiplier. For example, if $L$ is a partial differential operator with constant coefficients, then $L$ is a bilinear multiplier operator.

We next present a number of examples of bilinear pseudodifferential operators with symbols that satisfy conditions in the spirit of (2).

Motivated by the study of certain commutator operators, R. Coifman and Y. Meyer investigated boundedness properties in Lebesgue spaces of bilinear pseudodifferential operators. Conditions satisfied by the symbols included the following types: for $x$-independent $\sigma$,

$$
\left|\partial_{\xi}^{\beta} \partial_{\eta}^{\gamma} \sigma(\xi, \eta)\right| \leq C_{\beta, \gamma}(|\xi|+|\eta|)^{-(|\beta|+|\gamma|)}
$$

or, for $x$-dependent $\sigma$,

$$
\left|\partial_{x}^{\alpha} \partial_{\xi}^{\beta} \partial_{\eta}^{\gamma} \sigma(x, \xi, \eta)\right| \leq C_{\alpha, \beta, \gamma}(1+|\xi|+|\eta|)^{-(|\beta|+|\gamma|)}
$$

or, for $x$-dependent $\sigma$ and some $0 \leq \rho \leq 1$,

$$
\left|\partial_{x}^{\alpha} \partial_{\xi}^{\beta} \partial_{\eta}^{\gamma} \sigma(x, \xi, \eta)\right| \leq C_{\alpha, \beta, \gamma}(1+|\xi|+|\eta|)^{\rho|\alpha|-\rho(|\beta|+|\gamma|)}
$$

for every $x, \xi, \eta \in \mathbb{R}^{n}$ and $\alpha, \beta, \gamma \in \mathbb{N}_{0}^{n}$. Thus, comparing with (2), when taking a derivative of $\sigma$ with respect to a component of the frequency variables $\xi$ or $\eta$, the powers on the right-hand sides of (4) and (5) decrease by one while the power on the right-hand side of (6) decreases by $\rho$; on the other hand, when taking a derivative of $\sigma$ with respect to a component of the space variable $x$, the power on the right-hand side of (5) stays unchanged while the power on the right-hand side of (6) increases by $\rho$.

The building blocks of operators with symbols satisfying (4) are the so-called paraproducts, whose use emerged with the works of J. M. Bony and of R. Coifman and Y. Meyer on paradifferential operators. The paraproduct of $f$ and $g$ is defined as

$$
\Pi(f, g)=\sum_{j=-\infty}^{\infty} \Delta_{j} f S_{j-3} g,
$$

where $S_{j}$ and $\Delta_{j}$ are Littlewood-Paley operators, with $S_{j}$ capturing frequency components of its argument up to the order of $2^{j}$ and $\Delta_{j}$ capturing frequency components of its argument of the order of $2^{j}$. More precisely,

$$
\begin{aligned}
& \widehat{S_{j}(f)}(\xi)=\varphi\left(2^{-j} \xi\right) \widehat{f}(\xi), \\
& \widehat{\Delta_{j}(f)}(\xi)=\psi\left(2^{-j} \xi\right) \hat{f}(\xi),
\end{aligned}
$$

where $\varphi$ is an infinitely differentiable function on $\mathbb{R}^{n}$ such that $\varphi(\xi)=1$ if $|\xi| \leq 1, \varphi(\xi)=0$ if $|\xi| \geq 2$, and $\psi(\xi)=$ $\varphi(\xi)-\varphi(2 \xi)$; see Figure 1. It follows that the product of two functions $f$ and $g$ defined on $\mathbb{R}^{n}$ can be decomposed as

$$
f g=\Pi(f, g)+\Pi(g, f)+\sum_{j=-\infty}^{\infty} \Delta_{j} f \sum_{\ell=-2}^{2} \Delta_{j+\ell} g .
$$

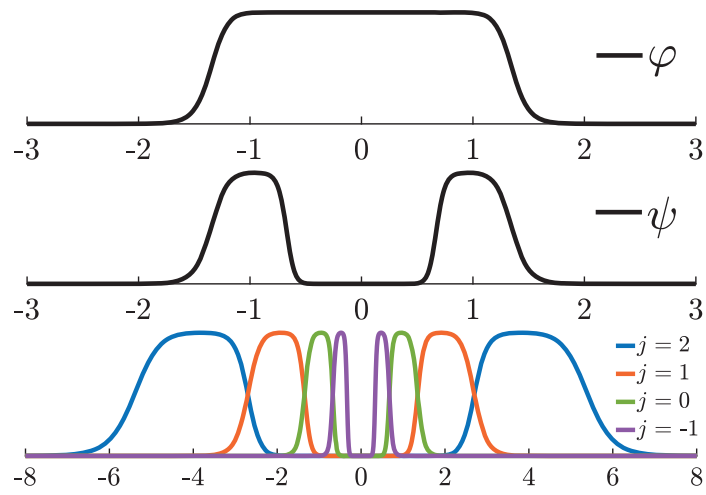

Figure 1. The functions $\varphi, \psi$, and $\psi\left(2^{-j} x\right)$.

Note that the $j$ th term in the paraproduct $\Pi$ given in (7) only contributes with frequency components of the order of $2^{j}$, since the support of its Fourier transform is contained in the set $\left\{\xi: 2^{j-2} \leq|\xi| \leq 2^{j+2}\right\}$; simple computations show that $\Pi$ is an operator of the type (3) with symbol $\sum_{j=-\infty}^{\infty} \psi\left(2^{-j} \xi\right) \varphi\left(2^{-j+3} \eta\right)$, which satisfies (4). Moreover, it can be proved that any operator (3) with symbol satisfying (4) can be written as a superposition of paraproduct-type operators; more precisely, it is the sum of an operator of the form

$$
\int_{\mathbb{R}^{2 n}} \sum_{j \in \mathbb{Z}} m_{j}(u, v) \Delta_{j}^{u} f(x) S_{j}^{v} g(x) \frac{d u d v}{\left(1+|u|^{2}+|v|^{2}\right)^{N}}
$$

and a similar one with the roles of $f$ and $g$ interchanged, where $\Delta_{j}^{u}$ and $S_{j}^{v}$ are appropriate Littlewood-Paley operators and $\sup _{j \in \mathbb{Z}, u, v \in \mathbb{R}^{n}}\left|m_{j}(u, v)\right|<\infty$.

Several different flavors of paraproduct operators, adapted to a variety of applications, have appeared since 
their introduction; the reader is referred for more details to the survey "What is ... a paraproduct?" (Notices Amer. Math. Soc. 57 (2010), no. 7, 858-860) by A. Bényi, D. Maldonado, and the author.

Operators with symbols satisfying estimates of the type (4) or (5) are also essential in the study of bilinear estimates known as fractional Leibniz rules. In turn, these stand as important tools for results on local and global wellposedness of nonlinear partial differential equations such as Euler, Navier-Stokes (see T. Kato and G. Ponce [KP88]) and Korteweg-de Vries, as well as in the study of smoothing properties of Schrödinger semigroups.

Fractional Leibniz rules in the setting of Lebesgue spaces for functions in $\mathbb{R}^{n}$ state that for $1 \leq p_{1}, p_{2} \leq \infty$ and $1 / 2 \leq p \leq \infty$ satisfying $1 / p=1 / p_{1}+1 / p_{2}$ and for $s>\max \{0, n(1 / p-1)\}$ or $s \in 2 \mathbb{N}$, it holds that

$$
\begin{aligned}
\left\|D^{S}(f g)\right\|_{L^{p}} & \lesssim\left\|D^{S} f\right\|_{L^{p_{1}}}\|g\|_{L^{p_{2}}}+\|f\|_{L^{p_{1}}}\left\|D^{S} g\right\|_{L^{p_{2}}}, \\
\left\|J^{S}(f g)\right\|_{L^{p}} & \lesssim\left\|J^{S} f\right\|_{L^{p_{1}}}\|g\|_{L^{p_{2}}}+\|f\|_{L^{p_{1}}}\left\|J^{S} g\right\|_{L^{p_{2}}},
\end{aligned}
$$

where $\|h\|_{L^{p}}=\left(\int_{\mathbb{R}^{n}}|h(x)|^{p} d x\right)^{1 / p}$ and the operators $D^{S}$ and $J^{S}$ are the homogeneous and inhomogeneous fractional differentiation operators of order $s \geq 0$, respectively, given by

$$
\begin{aligned}
& \widehat{D^{s} f}(\xi)=|\xi|^{s} \widehat{f}(\xi), \quad \xi \in \mathbb{R}^{n}, \\
& \widehat{J^{s} f}(\xi)=\left(1+|\xi|^{2}\right)^{\frac{s}{2}} \widehat{f}(\xi), \quad \xi \in \mathbb{R}^{n} .
\end{aligned}
$$

$D^{s}$ can be thought of as an operator that takes derivatives of order $s$ of its argument while $J^{s}$ can be interpreted as an operator that takes derivatives up to order $s$ of its argument; therefore, the estimates (8) and (9) are reminiscent of the Leibniz rule for the derivative of a product of functions taught in the calculus courses.

As it turns out, operators with symbols satisfying (4) or (5) constitute building blocks for the bilinear operators $(f, g) \mapsto D^{S}(f g)$ and $(f, g) \mapsto J^{S}(f g)$, respectively. More precisely, a decomposition in terms of frequency variables leads to a representation for $J^{S}(f g)$ given by

$$
J^{s}(f g)=T_{\sigma_{1}}\left(J^{s} f, g\right)+T_{\sigma_{2}}\left(f, J^{s} g\right)+T_{\sigma_{3}}\left(J^{s} f, g\right),
$$

where $\sigma_{1}$ and $\sigma_{2}$ satisfy (5) while $\sigma_{3}$ verifies (5) for $s$ sufficiently large. Consequently, boundedness properties in Lebesgue spaces of the form

$$
\left\|T_{\sigma_{j}}(f, g)\right\|_{L^{p}} \lesssim\|f\|_{L^{p_{1}}}\|g\|_{L^{p_{2}}}, \quad j=1,2,3,
$$

lead to the right-hand side of (9). An analogous argument, now involving the condition (4), applies to $D^{S}(f g)$.

Numerous works have recently emerged showcasing the connections between operators with symbols satisfying (4) or (5) and related fractional Leibniz-type rules in various function spaces; see Á. Bényi and R. H. Torres [BT03],
L. Grafakos and S. Oh [GO14], A. Thomson and the author [NT19b], R. H. Torres [Tor20], and the references therein.

Operators with symbols satisfying estimates of the type (5) or (6) also play a role in the proofs of boundedness properties of commutators. More precisely, given a linear pseudodifferential operator $T$ and a function $A$, the commutator of $T$ and the operator $M_{A}$ corresponding to pointwise multiplication by $A$ is defined by $\left[T, M_{A}\right](f)=$ $T(A f)-A T(f)$. Assuming that $A$ is a Lipschitz function in $\mathbb{R}^{n}$ (that is, $|A(x)-A(y)| \lesssim|x-y|$ for all $x, y \in \mathbb{R}^{n}$ ) and the symbol of $T$ satisfies appropriate conditions, R. Coifman and Y. Meyer [CM78] proved $L^{p}$ estimates for $\left[T, M_{A}\right]$. Depending on the conditions assumed on the symbol of $T$, the proof of these results involves the decomposition of the commutator into pieces that include bilinear pseudodifferential operators with symbols satisfying (5) or (6).

After the foundational work of R. Coifman and Y. Meyer, the field of bilinear (and multilinear) Fourier analysis experienced a rapid growth with highlights that include the study of boundedness properties of the bilinear Hilbert transform in Lebesgue spaces by M. Lacey and $\mathrm{C}$. Thiele, the further development of the bilinear Calderón-Zygmund theory by M. Christ and J.-L. Journé, C. Kenig and E. Stein, and L. Grafakos and R. H. Torres [GT02], as well as the first works regarding bilinear Hörmander classes by Á. Bényi and R. H. Torres [BT03, BT04].

Spurred by [BT03, BT04], significant efforts have been dedicated in recent years to the study of bilinear pseudodifferential operators with symbols in classes that unify conditions (2), (5), and (6). Namely, given $m \in \mathbb{R}$ and $0 \leq \delta \leq \rho \leq 1$, the bilinear Hörmander class $B S_{\rho, \delta}^{m}$ consists of symbols $\sigma(x, \xi, \eta)$ satisfying

$$
\left|\partial_{x}^{\alpha} \partial_{\xi}^{\beta} \partial_{\eta}^{\gamma} \sigma(x, \xi, \eta)\right| \leq C_{\alpha \beta \gamma}(1+|\xi|+|\eta|)^{m+\delta|\alpha|-\rho(|\beta|+|\gamma|)}
$$

for all $x, \xi, \eta \in \mathbb{R}^{n}$, for all multi-indices $\alpha, \beta, \gamma \in \mathbb{N}_{0}^{n}$, and for some constants $C_{\alpha \beta \gamma}>0$. The index $m$ is referred to as the order of (the symbols in) the class $B S_{\rho, \delta}^{m}$. Hence, we have that $B S_{1,0}^{m}$ corresponds to symbols satisfying (2), $B S_{1,0}^{0}$ corresponds to symbols satisfying (5), and $B S_{\rho, \rho}^{0}$ corresponds to symbols satisfying (6).

Two fundamental aspects of the study of the bilinear Hörmander classes are the symbolic calculus for transposes of operators and mapping properties in a variety of settings that include Lebesgue and Hardy spaces, the space $\mathrm{BMO}$ of functions of bounded mean oscillations, and Besov and Triebel-Lizorkin spaces. The corresponding literature is vast and includes works by Á. Bényi and R. H. Torres [BT03, BT04], Á. Bényi, D. Maldonado, R. H. Torres, and the author [BMNT10], Á. Bényi, F. Bernicot, D. Maldonado, R. H. Torres, and the author $\left[\mathrm{BBM}^{+} 13\right]$, A. Miyachi and N. Tomita [MT13], N. Michalowski, D. Rule, and W. Staubach [MRS14], the 
author [Nai15a, Nai15b], K. Koezuka and N. Tomita [KT18], A. Miyachi and N. Tomita [MT20, MT19], A. Thomson and the author [NT19a], and the references therein.

The classes $B S_{\rho, \delta}^{m}$ stand as the bilinear counterparts of the Hörmander classes $S_{\rho, \delta}^{m}$ associated to linear pseudodifferential operators. As we will see, while the bilinear and linear theories have some features in common, they require the use and development of different tools and techniques in several aspects.

\section{A Brief on the Linear Setting}

With earlier works by S. Mihlin, by A. Calderón and A. Zygmund, by A. Unterberger and J. Bokobza, and by R. Seeley, the theory of linear pseudodifferential operators was formalized in the 1960 s by J. Kohn and L. Nirenberg and by L. Hörmander. These operators, in particular those with symbols in the Hörmander classes $S_{\rho, \delta}^{m}$ defined below, have played a central role in the analysis of partial differential equations.

In the linear setting, a pseudodifferential operator is associated to a symbol $\sigma(x, \xi)$ defined for $x, \xi \in \mathbb{R}^{n}$ and is given by

$$
T_{\sigma} f(x)=\int_{\mathbb{R}^{n}} \sigma(x, \xi) \widehat{f}(\xi) e^{2 \pi i \xi \cdot x} d \xi .
$$

For $m \in \mathbb{R}$ and $0 \leq \delta \leq \rho \leq 1$, the Hörmander class $S_{\rho, \delta}^{m}$ is defined as the family of symbols $\sigma: \mathbb{R}^{n} \times \mathbb{R}^{n} \rightarrow \mathbb{C}$ such that

$$
\left|\partial_{x}^{\alpha} \partial_{\xi}^{\beta} \sigma(x, \xi)\right| \leq C_{\alpha \beta}(1+|\xi|)^{m+\delta|\alpha|-\rho|\beta|}
$$

for all $x, \xi \in \mathbb{R}^{n}$ and all multi-indices $\alpha, \beta \in \mathbb{N}_{0}^{n}$.

The study of boundedness properties of pseudodifferential operators with symbols in $S_{\rho, \delta}^{m}$ in the setting of Lebesgue spaces goes back to the 1960s and 1970s with the works of I. Hirschman, S. Wainger, L. Hörmander, A. Calderón and R. Vaillancourt, and C. Fefferman and E. Stein.

A seminal result in this direction is due to A. Calderón and R. Vaillancourt [CV72], who established that symbols in the class $S_{\rho, \rho}^{0}$ for $0 \leq \rho<1$ (that is, symbols of order zero) give rise to pseudodifferential operators that are bounded on $L^{2}\left(\mathbb{R}^{n}\right)$. Due to the nested properties of the Hörmander classes, this implies boundedness on $L^{2}\left(\mathbb{R}^{n}\right)$ for the operators with symbols in the classes $S_{\rho, \delta}^{0}$ with $0 \leq \delta<\rho<1$, previously proved by L. Hörmander [Hör67]. In turn, the boundedness of operators with symbols of order zero plays a significant role in the proof of endpoint boundedness properties for pseudodifferential operators with symbols in other Hörmander classes (C. Fefferman [Fef73]). More precisely, the following result holds.

Theorem 1. Let $0 \leq \delta \leq \rho \leq 1,0 \leq \delta<1$, and $1 \leq p \leq \infty$; define $m_{\rho}(p)=-n(1-\rho)\left|\frac{1}{p}-\frac{1}{2}\right|$.
1. If $1<p<\infty$ and $m \leq m_{\rho}(p)$, then $T_{\sigma}$ is bounded from $L^{p}\left(\mathbb{R}^{n}\right)$ into $L^{p}\left(\mathbb{R}^{n}\right)$ for all $\sigma \in S_{\rho, \delta}^{m}$.

2. If $p=1$ and $m \leq m_{\rho}(1)=-n(1-\rho) / 2$, then $T_{\sigma}$ is bounded from $H^{1}\left(\mathbb{R}^{n}\right)$ into $L^{1}\left(\mathbb{R}^{n}\right)$ for all $\sigma \in S_{\rho, \delta}^{m}$.

3. If $p=\infty$ and $m \leq m_{\rho}(\infty)=-n(1-\rho) / 2$, then $T_{\sigma}$ is bounded from $L^{\infty}\left(\mathbb{R}^{n}\right)$ into $B M O\left(\mathbb{R}^{n}\right)$ for all $\sigma \in S_{\rho, \delta}^{m}$.

4. If $1<p<\infty, 0<\rho<1$, and $m>m_{\rho}(p)$, there exists $\sigma \in S_{\rho, \delta}^{m}$ such that $T_{\sigma}$ fails to be bounded from $L^{p}\left(\mathbb{R}^{n}\right)$ into $L^{p}\left(\mathbb{R}^{n}\right)$.

The space $H^{1}\left(\mathbb{R}^{n}\right)$ is the Hardy space (defined on page 1127), which is contained in $L^{1}\left(\mathbb{R}^{n}\right)$, and the space $B M O\left(\mathbb{R}^{n}\right)$ is the space of functions of bounded mean oscillation (modulo constants), which contains $L^{\infty}\left(\mathbb{R}^{n}\right)$. Both $H^{1}\left(\mathbb{R}^{n}\right)$ and $B M O\left(\mathbb{R}^{n}\right)$ are natural substitutions for $L^{1}\left(\mathbb{R}^{n}\right)$ as a domain and $L^{\infty}\left(\mathbb{R}^{n}\right)$ as a codomain, respectively, when studying endpoint boundedness properties corresponding to $p=1$ and $p=\infty$. In view of parts 1 and 4 of Theorem 1 , $m_{\rho}(p)$ is called a critical order.

Homogeneous $x$-independent versions of the symbols in the class $S_{1,0}^{0}$ are given by multipliers satisfying Mihlin's condition, a version of (4) in $\mathbb{R}^{n}$; that is, multipliers $\sigma=$ $\sigma(\xi)$ for which

$$
\left|\partial^{\beta} \sigma(\xi)\right| \leq C_{\beta}|\xi|^{-|\beta|}
$$

up to a certain number of derivatives $|\beta|$. This condition clearly holds for symbols in $S_{1,0}^{0}$ that are $x$-independent. It turns out that if (12) holds for $|\beta| \leq[n / 2]+1$, then $T_{\sigma}$ has the same boundedness properties in Lebesgue spaces as stated for $S_{1,0}^{0}$.

The systematic study of linear pseudodifferential operators with symbols in the Hörmander classes includes a symbolic calculus (which plays a pivotal role in the treatment of approximate inverses of partial differential operators), connections to the Calderón-Zygmund theory of singular integrals, and boundedness properties in various function spaces.

The linear and bilinear settings for pseudodifferential operators with symbols in the Hörmander classes share similarities regarding the symbolic calculus as well as their connections to the Calderón-Zygmund theory; we will also see that the theories present different features when it comes to boundedness properties in the setting of Lebesgue spaces. Let us now, and for the rest of the article, focus on the main aspects of the bilinear theory.

\section{Bilinear Symbolic Calculus and Interpolation}

Our first goal will be to illustrate how a symbolic calculus associated to the bilinear Hörmander classes and interpolation play a central role for proving boundedness properties of corresponding bilinear pseudodifferential operators.

A bilinear pseudodifferential operator $T_{\sigma}$ is linear in each argument and, by "freezing" one of them at a time, 
has two transposes; more precisely, the first and second transposes of $T_{\sigma}$ are denoted $T_{\sigma}^{* 1}$ and $T_{\sigma}^{* 2}$, respectively, and are defined by

$$
\begin{aligned}
& \left\langle T_{\sigma}^{* 1}(f, g), h\right\rangle=\left\langle T_{\sigma}(h, g), f\right\rangle, \\
& \left\langle T_{\sigma}^{* 2}(f, g), h\right\rangle=\left\langle T_{\sigma}(f, h), g\right\rangle .
\end{aligned}
$$

Given $\sigma \in B S_{\rho, \delta}^{m}$, a natural question to ask is whether $T_{\sigma}^{* 1}$ and $T_{\sigma}^{* 2}$ are bilinear pseudodifferential operators and, in that case, what can be said about their symbols. The answers to these questions were provided by Á. Bényi and R. H. Torres [BT03] and by Á. Bényi, D. Maldonado, R. H. Torres, and the author [BMNT10], who showed that the bilinear Hörmander classes are closed under transposition. That is, if $m \in \mathbb{R}, 0 \leq \delta \leq \rho \leq 1$, and $\delta<1$, it holds that

$$
\begin{gathered}
\sigma \in B S_{\rho, \delta}^{m} \\
\Downarrow
\end{gathered}
$$

$$
T_{\sigma}^{* 1} \text { and } T_{\sigma}^{* 2} \text { have symbols in } B S_{\rho, \delta}^{m} \text {. }
$$

The importance of this result lies in the following observations. Suppose that $\sigma \in B S_{\rho, \delta}^{m}$ and fix $1 \leq p_{1} \leq \infty$, $1 \leq p_{2} \leq \infty$, and $1 \leq p \leq \infty$; then, by duality arguments it follows that

$$
\begin{gathered}
T_{\sigma}: L^{p_{1}}\left(\mathbb{R}^{n}\right) \times L^{p_{2}}\left(\mathbb{R}^{n}\right) \rightarrow L^{p}\left(\mathbb{R}^{n}\right) \\
\Downarrow \\
T_{\sigma}^{* 1}: L^{p^{\prime}}\left(\mathbb{R}^{n}\right) \times L^{p_{2}}\left(\mathbb{R}^{n}\right) \rightarrow L^{p_{1}^{\prime}}\left(\mathbb{R}^{n}\right) \\
T_{\sigma}^{* 2}: L^{p_{1}}\left(\mathbb{R}^{n}\right) \times L^{p^{\prime}}\left(\mathbb{R}^{n}\right) \rightarrow L^{p_{2}^{\prime}}\left(\mathbb{R}^{n}\right),
\end{gathered}
$$

where the notation $T_{\sigma}: X \times Y \rightarrow Z$ means that $T_{\sigma}$ is bounded from $X \times Y$ into $Z$ and, for $1 \leq q \leq \infty$, $q^{\prime}$ denotes the conjugate exponent of $q$, that is, $1 / q+$ $1 / q^{\prime}=1$. In view of (13) and (14) along with the fact that $\left(T_{\sigma}^{* j}\right)^{* j}=T_{\sigma}$ for $j=1,2$, it follows that if every operator $T_{\sigma}$ with $\sigma \in B S_{\rho, \delta}^{m}$ is bounded from $L^{p_{1}}\left(\mathbb{R}^{n}\right) \times$ $L^{p_{2}}\left(\mathbb{R}^{n}\right)$ into $L^{p}\left(\mathbb{R}^{n}\right)$, then every operator $T_{\sigma}$ with $\sigma \in$ $B S_{\rho, \delta}^{m}$ is also bounded from $L^{p^{\prime}}\left(\mathbb{R}^{n}\right) \times L^{p_{2}}\left(\mathbb{R}^{n}\right)$ into $L^{p_{1}^{\prime}}\left(\mathbb{R}^{n}\right)$ and from $L^{p_{1}}\left(\mathbb{R}^{n}\right) \times L^{p^{\prime}}\left(\mathbb{R}^{n}\right)$ into $L^{p_{2}^{\prime}}\left(\mathbb{R}^{n}\right)$. In turn, bilinear interpolation implies that every such operator $T_{\sigma}$ is bounded from $L^{q_{1}}\left(\mathbb{R}^{n}\right) \times L^{q_{2}}\left(\mathbb{R}^{n}\right)$ into $L^{q}\left(\mathbb{R}^{n}\right)$ for every $q_{1}, q_{2}$, and $q$ such that $\left(1 / q_{1}, 1 / q_{2}, 1 / q\right)$ lies in the triangle with vertices $\left(1 / p_{1}, 1 / p_{2}, 1 / p\right),\left(1 / p^{\prime}, 1 / p_{2}, 1 / p_{1}^{\prime}\right)$, and $\left(1 / p_{1}, 1 / p^{\prime}, 1 / p_{2}^{\prime}\right)$. See Figure 2 .

Next let us describe how complex interpolation can be used for proving boundedness properties of bilinear pseudodifferential operators involving different Hörmander classes.

Note that the bilinear Hörmander classes can be given the structure of a Fréchet space. Indeed, for $\sigma \in B S_{\rho, \delta}^{m}$ and

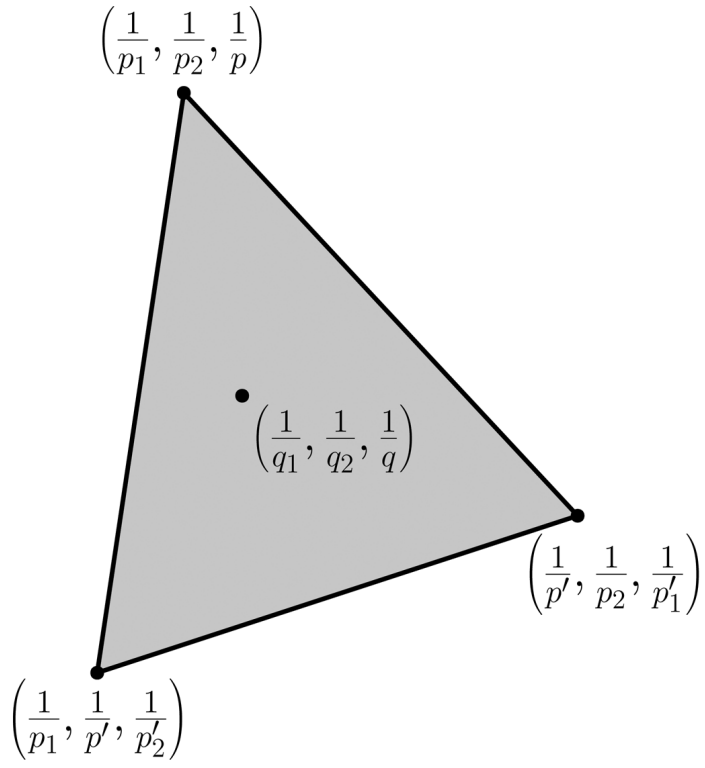

Figure 2. Boundedness from $L^{p_{1}}\left(\mathbb{R}^{n}\right) \times L^{p_{2}}\left(\mathbb{R}^{n}\right)$ into $L^{p}\left(\mathbb{R}^{n}\right)$ for all $T_{\sigma}$ with $\sigma \in B S_{\rho, \delta}^{m}$ implies boundedness from $L^{q_{1}}\left(\mathbb{R}^{n}\right) \times L^{q_{2}}\left(\mathbb{R}^{n}\right)$ into $L^{q}\left(\mathbb{R}^{n}\right)$ for all $T_{\sigma}$ with $\sigma \in B S_{\rho, \delta}^{m}$.

$N \in \mathbb{N}_{0}$, define

$$
\|\sigma\|_{N}=\sup _{\substack{|\alpha| \leq N \\|\beta|,|\gamma| \leq N}} \sup _{x, \xi, \eta \in \mathbb{R}^{n}} \frac{\left|\partial_{x}^{\alpha} \partial_{\xi}^{\beta} \partial_{\eta}^{\gamma} \sigma(x, \xi, \eta)\right|}{(1+|\xi|+|\eta|)^{m+\delta|\alpha|-\rho(|\beta|+|\gamma|)}} .
$$

Then $B S_{\rho, \delta}^{m}$ is a Fréchet space with the topology induced by the family of norms $\left\{\|\cdot\|_{N}\right\}_{N \in \mathbb{N}_{0}}$.

For $\sigma \in B S_{\rho, \delta}^{m}$, consider the trilinear operator $T$ given by

$$
T(\sigma, f, g):=T_{\sigma}(f, g) .
$$

The notation

$$
T: B S_{\rho, \delta}^{m} \times X \times Y \rightarrow Z,
$$

where $X, Y$, and $Z$ are function spaces, will express the fact that $T$ maps continuously from $B S_{\rho, \delta}^{m} \times X \times Y$ into $Z$, that is, there exists $N \in \mathbb{N}_{0}$ such that

$$
\|T(\sigma, f, g)\|_{Z} \lesssim\|\sigma\|_{N}\|f\|_{X}\|g\|_{Y}
$$

for all $\sigma \in B S_{\rho, \delta}^{m}, f \in X$, and $g \in Y$.

It turns out that, given $0 \leq \rho<1, m_{0}, m_{1} \in \mathbb{R}$, and $p_{1}, p_{2}, p, \widetilde{p}_{1}, \widetilde{p}_{2}, \widetilde{p} \in[1, \infty]$, complex interpolation leads to

$$
\begin{gathered}
T: B S_{\rho, \rho}^{m_{0}} \times L^{p_{1}}\left(\mathbb{R}^{n}\right) \times L^{p_{2}}\left(\mathbb{R}^{n}\right) \rightarrow L^{p}\left(\mathbb{R}^{n}\right) \\
T: B S_{\rho, \rho}^{m_{1}} \times L^{\widetilde{p}_{1}}\left(\mathbb{R}^{n}\right) \times L^{\widetilde{p}_{2}}\left(\mathbb{R}^{n}\right) \rightarrow L^{\widetilde{p}}\left(\mathbb{R}^{n}\right) \\
\Downarrow \\
T: B S_{\rho, \rho}^{m} \times L^{q_{1}}\left(\mathbb{R}^{n}\right) \times L^{q_{2}}\left(\mathbb{R}^{n}\right) \rightarrow L^{q}\left(\mathbb{R}^{n}\right),
\end{gathered}
$$

where, for some $0<\theta<1$,

$$
\begin{aligned}
\left(\frac{1}{q_{1}}, \frac{1}{q_{2}}, \frac{1}{q}\right) & =(1-\theta)\left(\frac{1}{p_{1}}, \frac{1}{p_{2}}, \frac{1}{p}\right)+\theta\left(\frac{1}{\widetilde{p}_{1}}, \frac{1}{\widetilde{p}_{2}}, \frac{1}{\widetilde{p}}\right), \\
m & =(1-\theta) m_{0}+\theta m_{1} .
\end{aligned}
$$




\section{Bilinear Kernel Estimates}

Before addressing the complete picture for the boundedness in Lebesgue spaces of bilinear pseudodifferential operators with symbols in the bilinear Hörmander classes, let us comment on the connection between $B S_{\rho, \delta}^{m}$ and the bilinear Calderón-Zygmund theory. Some boundedness properties in Lebesgue spaces will be deduced from this relation.

A bilinear pseudodifferential operator may also be represented in the form

$$
T_{\sigma}(f, g)(x)=\int_{\mathbb{R}^{2 n}} K_{\sigma}(x, y, z) f(y) g(z) d y d z,
$$

where $K_{\sigma}$ is called the bilinear kernel of $T_{\sigma}$. This follows from expressing $\widehat{f}$ and $\hat{g}$ in (3) in terms of $f$ and $g$ and formally interchanging the order of integration to get that $K_{\sigma}$ is given by the oscillatory integral

$$
K_{\sigma}(x, y, z)=\int_{\mathbb{R}^{2 n}} \sigma(x, \xi, \eta) e^{2 \pi i \xi \cdot(x-y)} e^{2 \pi i \eta \cdot(x-z)} d \xi d \eta .
$$

For $\sigma$ in the Hörmander classes $B S_{\rho, \delta}^{m}$, the kernel $K_{\sigma}$ and its partial derivatives are fast decreasing away from the diagonal in $\mathbb{R}^{3 n}, \Delta=\left\{(x, x, x): x \in \mathbb{R}^{n}\right\}$, in the sense that given $\alpha, \beta, \gamma \in \mathbb{N}_{0}^{n}$ and $N$ sufficiently large, it holds that

$$
\left|\partial_{x}^{\alpha} \partial_{y}^{\beta} \partial_{z}^{\gamma} K_{\sigma}(x, y, z)\right| \lesssim \frac{1}{(|x-y|+|x-z|+|y-z|)^{N}} .
$$

Any singularities of the kernel $K_{\sigma}$ are contained in the diagonal $\Delta$ and the following estimates hold: if $m+M+2 n>0$ for some $M \in \mathbb{N}_{0}$, then

$$
\begin{aligned}
\left|\partial_{x}^{\alpha} \partial_{y}^{\beta} \partial_{z}^{\gamma} K_{\sigma}(x, y, z)\right| & 1 \\
& \lesssim \frac{1}{(|x-y|+|x-z|+|y-z|)^{\frac{m+M+2 n}{\rho}}}
\end{aligned}
$$

for all $x, y, z \in \mathbb{R}^{n}$ with $(x, y, z) \notin \Delta$ and for $\alpha, \beta, \gamma \in \mathbb{N}_{0}$ such that $|\alpha+\beta+\gamma|=M$. The above estimates along with other ones were proved by Á. Bényi, D. Maldonado, R. H. Torres, and the author [BMNT10].

Such kernel estimates allow for a first glance at boundedness properties in the context of Lebesgue spaces of bilinear pseudodifferential operators with symbols in some, but not all, of the bilinear Hörmander classes by way of the bilinear Calderón-Zygmund theory. We first note that the symbol $\tilde{\sigma} \equiv 1$ belongs to $B S_{\rho, \delta}^{m}$ for $m \geq 0$ and any $\rho$ and $\delta$ and, given that $T_{\tilde{\sigma}}(f, g)=f g$, by Hölder's inequality, we have

$$
\left\|T_{\tilde{\sigma}}(f, g)\right\|_{L^{p}} \leq\|f\|_{L^{p_{1}}}\|g\|_{L^{p_{2}}}
$$

for $0<p_{1} \leq \infty, 0<p_{2} \leq \infty$, and $0<p \leq \infty$ such that

$$
\frac{1}{p}=\frac{1}{p_{1}}+\frac{1}{p_{2}} \text {. }
$$

As a consequence, the Hölder condition (17) arises as a natural one to require when studying continuity properties of bilinear pseudodifferential operators from $L^{p_{1}}\left(\mathbb{R}^{n}\right) \times$ $L^{p_{2}}\left(\mathbb{R}^{n}\right)$ into $L^{p}\left(\mathbb{R}^{n}\right)$.

A bilinear Calderón-Zygmund operator is a bilinear operator that is bounded from $L^{2}\left(\mathbb{R}^{n}\right) \times L^{2}\left(\mathbb{R}^{n}\right)$ into $L^{1}\left(\mathbb{R}^{n}\right)$ and satisfies

$$
T(f, g)(x)=\int_{\mathbb{R}^{2 n}} K(x, y, z) f(y) g(z) d y d z
$$

for $f, g \in C_{0}^{\infty}\left(\mathbb{R}^{n}\right)$ and for all $x \notin \operatorname{supp}(f) \cap \operatorname{supp}(g)$, where the kernel $K$ is locally integrable in $\mathbb{R}^{3 n} \backslash \Delta$ and satisfies the size condition

$$
|K(x, y, z)| \lesssim \frac{1}{(|x-y|+|x-z|+|y-z|)^{2 n}}
$$

for all $x, y, z \in \mathbb{R}^{n}$ such that $(x, y, z) \notin \Delta$, and the smoothness estimate

$$
\begin{aligned}
& \left|K(x, y, z)-K\left(x^{\prime}, y, z\right)\right| \\
& \quad \lesssim \frac{\left|x-x^{\prime}\right|^{\varepsilon}}{(|x-y|+|x-z|+|y-z|)^{2 n+\varepsilon}}
\end{aligned}
$$

for $\left|x-x^{\prime}\right| \leq \frac{1}{2} \max \{|x-y|,|x-z|,|y-z|\}$ and for some $\varepsilon>0$, along with analogous smoothness estimates corresponding to $y$ and $z$. A kernel satisfying (18), (19), and the corresponding smoothness estimates for $y$ and $z$ is called a Calderón-Zygmund kernel.

As proved by L. Grafakos and R. H. Torres [GT02], the bilinear Calderón-Zygmund theory establishes that bilinear Calderón-Zygmund operators can be extended to bounded operators from $L^{p_{1}}\left(\mathbb{R}^{n}\right) \times L^{p_{2}}\left(\mathbb{R}^{n}\right)$ into $L^{p}\left(\mathbb{R}^{n}\right)$ for $1<p_{1}<\infty, 1<p_{2}<\infty$, and $1 / 2<p<\infty$ satisfying the Hölder condition (17); moreover, they are bounded from $L^{1}\left(\mathbb{R}^{n}\right) \times L^{1}\left(\mathbb{R}^{n}\right)$ into the Lorentz space $L^{\frac{1}{2}, \infty}\left(\mathbb{R}^{n}\right)$, from $L^{1}\left(\mathbb{R}^{n}\right) \times L^{p_{2}}\left(\mathbb{R}^{n}\right)$ into the Lorentz space $L^{p, \infty}\left(\mathbb{R}^{n}\right)$ with $p_{1}=1,1<p_{2}<\infty$, and $1 / 2<p<\infty$ satisfying (17), from $L^{p_{1}}\left(\mathbb{R}^{n}\right) \times L^{1}\left(\mathbb{R}^{n}\right)$ into $L^{p, \infty}\left(\mathbb{R}^{n}\right)$ with $1<p_{1}<\infty$, $p_{2}=1$, and $1 / 2<p<\infty$ satisfying (17), and from $L^{\infty}\left(\mathbb{R}^{n}\right) \times L^{\infty}\left(\mathbb{R}^{n}\right)$ into $B M O\left(\mathbb{R}^{n}\right)$.

It follows from (16) that, for $\sigma \in B S_{1, \delta}^{0}$ with $0 \leq \delta \leq 1$, the kernel $K_{\sigma}$ of $T_{\sigma}$ satisfies the size estimate (18) as well as the estimate

$$
\left|\nabla K_{\sigma}(x, y, z)\right| \lesssim \frac{1}{(|x-y|+|x-z|+|y-z|)^{2 n+1}},
$$

which readily implies (19) and its counterparts for $y$ and $z$ with $\varepsilon=1$. Moreover, it turns out that operators with symbols in $B S_{1, \delta}^{0}$ are bilinear Calderón-Zygmund operators for any choice of $0 \leq \delta<1$; as such, these operators satisfy all boundedness properties mentioned above. On the other hand, operators with symbols in $B S_{1,1}^{0}$ have bilinear Calderón-Zygmund kernels but are not necessarily bounded on Lebesgue spaces. However, as shown by $\mathrm{L}$. Grafakos and R. H. Torres [GT02], if $\sigma \in B S_{1,1}^{0}$ and the transposes of $T_{\sigma}$ have symbols in $B S_{1,1}^{0}$, then $T_{\sigma}$ is a bilinear Calderón-Zygmund operator. 
By using a definition of Calderón-Zygmund operator that requires a weaker version of (19) and its analogues for $y$ and $z$, it follows that if $\sigma \in B S_{\rho, \delta}^{m}$ with $0 \leq \delta \leq \rho \leq 1$, $0<\delta<1,0<\rho \leq 1$, and $m<-2 n(1-\rho)$, then $T_{\sigma}$ is also a bilinear Calderón-Zygmund operator.

The realization of bilinear pseudodifferential operators with symbols in the Hörmander classes as bilinear Calderón-Zygmund operators to deduce boundedness properties is only effective in the situations discussed above. In general, the classes $B S_{\rho, \delta}^{m}$ give rise to operators that go beyond the bilinear Calderon-Zygmund theory and the study of their boundedness properties requires the development and implementation of novel techniques.

Finally, we mention that bilinear Coifman-Meyer multiplier operators (that is, pseudodifferential operators whose symbols satisfy (4)) also furnish examples of bilinear Calderón-Zygmund operators.

\section{Boundedness on Lebesgue Spaces}

As presented in Theorem 1, the critical order for $L^{p}$-boundedness for linear pseudodifferential operators with symbols in the linear Hörmander classes $S_{\rho, \delta}^{m}$ is $-n(1-\rho)|1 / p-1 / 2|$. Let us now discuss the critical order for bilinear pseudodifferential operators with symbols in the bilinear Hörmander classes to be bounded from $L^{p_{1}}\left(\mathbb{R}^{n}\right) \times L^{p_{2}}\left(\mathbb{R}^{n}\right)$ into $L^{p}\left(\mathbb{R}^{n}\right)$.

As opposed to what occurs in the linear theory, operators with symbols in the bilinear Hörmander classes of order zero need not be bounded in Lebesgue spaces. More precisely, given $0 \leq \delta \leq \rho<1,1 \leq p_{1}<\infty, 1 \leq p_{2}<\infty$, and $1 \leq p<\infty$ satisfying the Hölder condition (17), there are symbols in $B S_{\rho, \delta}^{0}$ that give rise to operators that are not bounded from $L^{p_{1}}\left(\mathbb{R}^{n}\right) \times L^{p_{2}}\left(\mathbb{R}^{n}\right)$ into $L^{p}\left(\mathbb{R}^{n}\right)$. This fact was proved for $B S_{0,0}^{0}$ by Á. Bényi and R. H. Torres [BT04] using $x$-independent symbols, while the general case follows from a scaling argument as shown by Á. Bényi, F. Bernicot, D. Maldonado, R. H. Torres, and the author [BBM $\left.{ }^{+} 13\right]$. This lack of boundedness ruled out the natural bilinear version of the Calderón-Vaillancourt result and sparked subsequent investigations regarding the critical orders for the bilinear Hörmander classes in the context of Lebesgue spaces.

It was proved by $\mathrm{N}$. Michalowski, D. Rule, and W. Staubach [MRS14] that if $1 \leq p_{1} \leq \infty, 1 \leq p_{2} \leq \infty$, and $1 \leq p \leq \infty$ satisfy (17), operators with symbols in $B S_{\rho, \delta}^{m}$, where $0 \leqslant \delta \leqslant \rho \leqslant 1$ and $\delta<1$, are bounded from $L^{p_{1}}\left(\mathbb{R}^{n}\right) \times L^{p_{2}}\left(\mathbb{R}^{n}\right)$ into $L^{p}\left(\mathbb{R}^{n}\right)$ provided that $m<$ $-n(1-\rho) \max \left\{\frac{1}{2},\left(\frac{2}{p_{1}}-\frac{1}{2}\right),\left(\frac{2}{p_{2}}-\frac{1}{2}\right),\left(\frac{3}{2}-\frac{2}{p}\right)\right\}$. This result was improved by Á. Bényi, F. Bernicot, D. Maldonado, R. H. Torres, and the author $\left[\mathrm{BBM}^{+} 13\right]$, who showed that the same boundedness holds true if $m<m_{\rho}\left(p_{1}, p_{2}\right)$ with

$$
m_{\rho}\left(p_{1}, p_{2}\right)=-n(1-\rho) \max \left\{\frac{1}{2}, \frac{1}{p_{1}}, \frac{1}{p_{2}}, \frac{1}{p^{\prime}}\right\}
$$

for $1 \leq p_{1} \leq \infty, 1 \leq p_{2} \leq \infty$, and $1 \leq p \leq \infty$ satisfisfying (17) and where $1 / p^{\prime}=1-1 / p$; see Figure 3 . On the other hand, if $m>m_{\rho}\left(p_{1}, p_{2}\right)$, then operators with symbols in the class $B S_{\rho, \delta}^{m}$ may not be bounded from $L^{p_{1}}\left(\mathbb{R}^{n}\right) \times L^{p_{2}}\left(\mathbb{R}^{n}\right)$ into $L^{p}\left(\mathbb{R}^{n}\right)$; this was proved by A. Miyachi and N. Tomita [MT13, MT20] along with the fact that boundedness does hold for operators with symbols of order $m_{\rho}\left(p_{1}, p_{2}\right)$. As a consequence, $m_{\rho}\left(p_{1}, p_{2}\right)$ is the critical order for all $\rho, p_{1}$, and $p_{2}$. The next theorem, which can be regarded as the bilinear counterpart of Theorem 1, summarizes all these results.

Theorem 2. Let $1 \leq p_{1} \leq \infty, 1 \leq p_{2} \leq \infty$, and $1 \leq p \leq \infty$ satisfy (17); consider $0 \leq \delta \leq \rho<1$.

1. If $m \leq m_{\rho}\left(p_{1}, p_{2}\right)$, all bilinear pseudodifferential operators with symbol in $B S_{\rho, \delta}^{m}$ are bounded from $L^{p_{1}}\left(\mathbb{R}^{n}\right) \times$ $L^{p_{2}}\left(\mathbb{R}^{n}\right)$ into $L^{p}\left(\mathbb{R}^{n}\right)$, where $L^{p_{1}}\left(\mathbb{R}^{n}\right)$ and $L^{p_{2}}\left(\mathbb{R}^{n}\right)$ should be replaced by the Hardy space $H^{1}\left(\mathbb{R}^{n}\right)$ if $p_{1}=1$ or $p_{2}=1$ and $L^{p}\left(\mathbb{R}^{n}\right)$ should be replaced by $B M O\left(\mathbb{R}^{n}\right)$ if $p=\infty$.

2. If $m>m_{\rho}\left(p_{1}, p_{2}\right)$, there are symbols in the class $B S_{\rho, \rho}^{m}$ for which the associated bilinear pseudodifferential operators are not bounded between the spaces as indicated in part 1.

Next, let us discuss the central steps in the proof of part 1 of Theorem 2. We first note that in view of the nesting properties of the bilinear Hörmander classes, we may assume that $\rho=\delta$ and that the order of the class is a critical order. Using the symbolic calculus and complex interpolation, it is enough to prove the cases $p_{1}=p_{2}=2($ then $p=1$

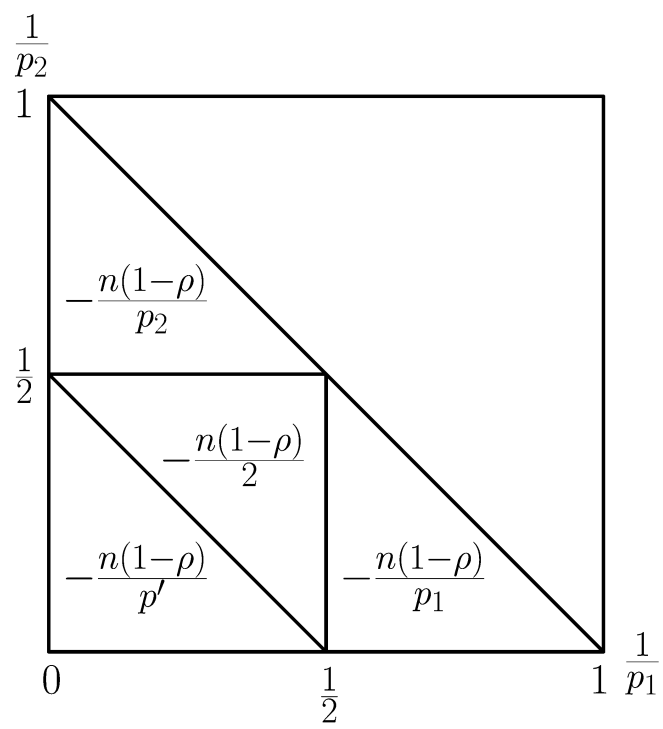

Figure 3. Visualization of $m_{\rho}\left(p_{1}, p_{2}\right)$ : for $\rho$ fixed, the diagram shows the values of $m_{\rho}\left(p_{1}, p_{2}\right)$ for $1 \leq p_{1} \leq \infty, 1 \leq p_{2} \leq \infty$, and $1 / p=1 / p_{1}+1 / p_{2}$. 
and $\left.m_{\rho}\left(p_{1}, p_{2}\right)=-n(1-\rho) / 2\right)$ and $p_{1}=p_{2}=\infty$ (then the target space is $B M O\left(\mathbb{R}^{n}\right)$ and $\left.m_{\rho}\left(p_{1}, p_{2}\right)=-n(1-\rho)\right)$. That is,

$$
\begin{aligned}
& T: B S_{\rho, \rho}^{-\frac{n}{2}(1-\rho)} \times L^{2}\left(\mathbb{R}^{n}\right) \times L^{2}\left(\mathbb{R}^{n}\right) \rightarrow L^{1}\left(\mathbb{R}^{n}\right) \\
& T: B S_{\rho, \rho}^{-n(1-\rho)} \times L^{\infty}\left(\mathbb{R}^{n}\right) \times L^{\infty}\left(\mathbb{R}^{n}\right) \rightarrow B M O\left(\mathbb{R}^{n}\right)
\end{aligned}
$$$$
\Downarrow
$$$$
T: B S_{\rho, \rho}^{m_{\rho}\left(p_{1}, p_{2}\right)} \times L^{p_{1}}\left(\mathbb{R}^{n}\right) \times L^{p_{2}}\left(\mathbb{R}^{n}\right) \rightarrow L^{p}\left(\mathbb{R}^{n}\right),
$$

where $p_{1}, p_{2}$, and $p$ are as in the statement of Theorem 2, $p \neq \infty, L^{p_{1}}\left(\mathbb{R}^{n}\right)$ and $L^{p_{2}}\left(\mathbb{R}^{n}\right)$ should be replaced by $H^{1}\left(\mathbb{R}^{n}\right)$ if $p_{1}=1$ or $p_{2}=1$, and $0 \leq \rho<1$.

Indeed, duality arguments, the symbolic calculus (13), and the boundedness (20) imply (22) for $p_{1}=2, p_{2}=\infty$ and $p_{1}=\infty, p_{2}=2$, for which $m_{\rho}\left(p_{1}, p_{2}\right)=-n(1-\rho) / 2$ (see Figure $4 \mathrm{~b}$ ):

$$
\begin{aligned}
& T: B S_{\rho, \rho}^{-\frac{n}{2}(1-\rho)} \times L^{2}\left(\mathbb{R}^{n}\right) \times L^{\infty}\left(\mathbb{R}^{n}\right) \rightarrow L^{2}\left(\mathbb{R}^{n}\right), \\
& T: B S_{\rho, \rho}^{-\frac{n}{2}(1-\rho)} \times L^{\infty}\left(\mathbb{R}^{n}\right) \times L^{2}\left(\mathbb{R}^{n}\right) \rightarrow L^{2}\left(\mathbb{R}^{n}\right) .
\end{aligned}
$$

Similarly, duality arguments, the symbolic calculus (13), and the boundedness (21) imply the result for $p_{1}=1, p_{2}=$ $\infty$ and $p_{1}=\infty, p_{2}=1$, for which $m_{\rho}\left(p_{1}, p_{2}\right)=-n(1-\rho)$ (see Figure $4 \mathrm{~b}$ ):

$$
\begin{aligned}
& T: B S_{\rho, \rho}^{-n(1-\rho)} \times H^{1}\left(\mathbb{R}^{n}\right) \times L^{\infty}\left(\mathbb{R}^{n}\right) \rightarrow L^{1}\left(\mathbb{R}^{n}\right), \\
& T: B S_{\rho, \rho}^{-n(1-\rho)} \times L^{\infty}\left(\mathbb{R}^{n}\right) \times H^{1}\left(\mathbb{R}^{n}\right) \rightarrow L^{1}\left(\mathbb{R}^{n}\right) .
\end{aligned}
$$

Complex interpolation as given in (15) then yields the result for $p_{1}$ and $p_{2}$ such that $\left(1 / p_{1}, 1 / p_{2}\right)$ is on the border of the triangle with vertices $(0,0),(0,1)$, and $(1,0)$. For instance, (25) and (20) imply (22) for $\left(1 / p_{1}, 1 / p_{2}\right)$ on the segment with endpoints $(1,0)$ and $(1 / 2,1 / 2)$ by noting that if $\left(1 / p_{1}, 1 / p_{2}\right)=(1-\theta)(1,0)+\theta(1 / 2,1 / 2)$ for some $0<\theta<1$, then $m_{\rho}\left(p_{1}, p_{2}\right)=(1-\theta) m_{\rho}(1,0)+\theta m_{\rho}(2,2)$; see Figure $4 \mathrm{c}$. The result for $p_{1}$ and $p_{2}$ such that $\left(1 / p_{1}, 1 / p_{2}\right)$ is in the interior of the triangle with vertices $(0,0),(1,0)$, and $(0,1)$ follows by bilinear complex interpolation since $m_{\rho}\left(p_{1}, p_{2}\right)$ is constant along horizontal segments in the triangle with vertices $(0,1),(0,1 / 2)$, and $(1 / 2,1 / 2) ; m_{\rho}\left(p_{1}, p_{2}\right)$ is constant along vertical segments in the triangle with vertices $(1,0)$, $(1 / 2,0)$, and $(1 / 2,1 / 2) ; m_{\rho}\left(p_{1}, p_{2}\right)$ is constant along diagonal segments in the triangle with vertices $(0,0),(0,1 / 2)$, and $(1 / 2,0)$; and $m_{\rho}\left(p_{1}, p_{2}\right)$ is constant in the triangle with vertices $(1 / 2,0),(0,1 / 2)$, and $(1 / 2,1 / 2)$. See Figure $4 \mathrm{~d}$.

A stronger result than the main estimate (21) was given by the author in [Nai15b] for $0<\rho<1 / 2$ in terms of pointwise inequalities for maximal operators: if $\sigma \in B S_{\rho, \delta}^{-n(1-\rho)}$ with $0 \leq \delta \leq \rho$ and $0<\rho<1 / 2$, the pointwise inequality

$$
\mathcal{M}^{\sharp}\left(T_{\sigma}(f, g)\right)(x) \lesssim\|\sigma\|_{N} \mathcal{M}_{2}(f)(x) \mathcal{M}_{2}(g)(x)
$$

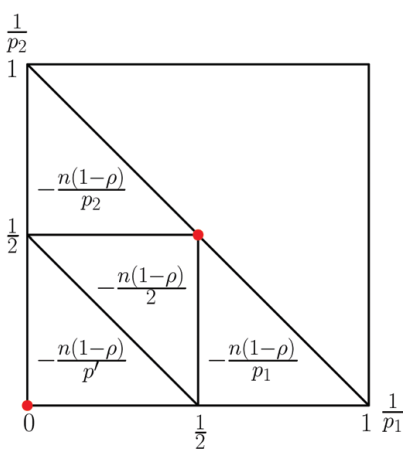

(a) Boundedness given in (20) $\left(p_{1}=p_{2}=2\right)$ and $(21)\left(p_{1}=p_{2}=\infty\right)$.

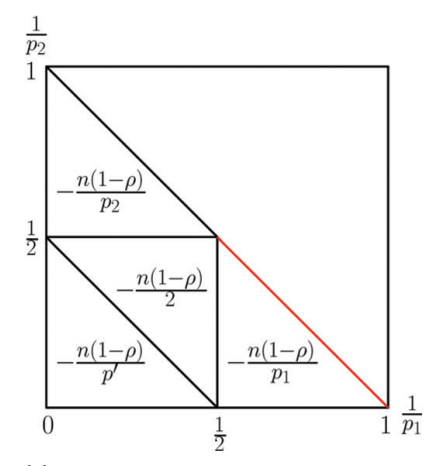

(c) From (20) and (25) through interpolation.

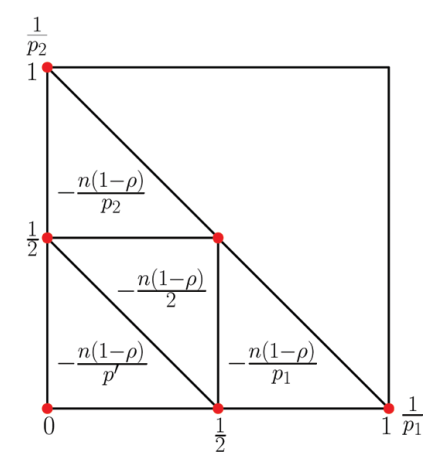

(b) From (20) and (21) through duality and symbolic calculus.

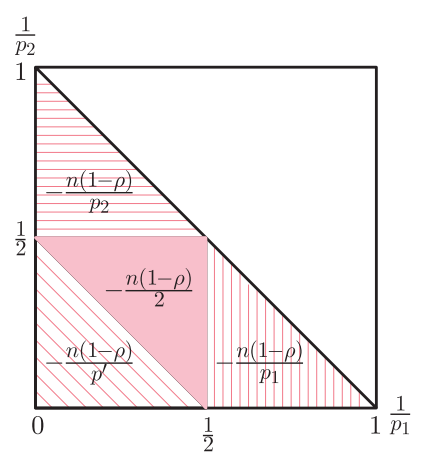

(d) From (22) at the border through interpolation.
Figure 4. Steps in the proof of part 1 of Theorem 2.

holds for some $N \in \mathbb{N}_{0}$ independent of $\sigma, f$, and $g$. Here $\mathcal{M}^{\sharp}$ is the sharp maximal operator defined as

$$
\mathcal{M}^{\sharp}(f)(x)=\sup _{x \in Q} \frac{1}{|Q|} \int_{Q}\left|f(y)-f_{Q}\right| d y,
$$

where the supremum is taken over all cubes $Q \subset \mathbb{R}^{n}$ that contain $x$ and $f_{Q}=\frac{1}{|Q|} \int_{Q} f(y) d y$, while $\mathcal{M}_{2}(f)=$ $\left(\mathcal{M}\left(|f|^{2}\right)\right)^{\frac{1}{2}}$ with $\mathcal{M}$ being the Hardy-Littlewood maximal operator given by

$$
\mathcal{M}(f)(x)=\sup _{x \in Q} \frac{1}{|Q|} \int_{Q}|f(y)| d y,
$$

where the supremum is taken over all cubes $Q \subset \mathbb{R}^{n}$ that contain $x$. Since $B M O\left(\mathbb{R}^{n}\right)$ is precisely the class of locally integrable functions $f$ (modulo constants) for which $\mathcal{M}^{\sharp} f \in L^{\infty}\left(\mathbb{R}^{n}\right)$ with $\|f\|_{B M O}=\left\|\mathcal{M}^{\sharp} f\right\|_{L^{\infty}}$ and $\mathcal{M}_{2}$ is clearly bounded on $L^{\infty}\left(\mathbb{R}^{n}\right)$, the estimate (21) readily follows from (27).

The proof of (27) relies on boundedness properties for bilinear pseudodifferential operators involving indices that do not satisfy the Hölder condition (17): if $0 \leq \delta \leq \rho$ and $0<\rho<1 / 2$, then

$$
T: B S_{\rho, \delta}^{-n(1-\rho)} \times L^{2}\left(\mathbb{R}^{n}\right) \times L^{2}\left(\mathbb{R}^{n}\right) \rightarrow L^{\frac{1}{\rho}}\left(\mathbb{R}^{n}\right) .
$$


In turn, (28) implies other continuity properties in Lebesgue spaces with off-Hölder indices for operators with symbols in $B S_{\rho, \delta}^{-n(1-\rho)}$; these follow from the symbolic calculus, duality, and interpolation.

The proof of (21) for the whole range $0<\rho<1$ was given by A. Miyachi and N. Tomita in [MT20] building upon the approach in [Nai15b] along with a novel use of certain characterizations of weak Lebesgue spaces. We refer the reader to [MT20] regarding the proof of (20) and part 2 of Theorem 2.

\section{Boundedness on Hardy Spaces}

The boundedness properties of bilinear pseudodifferential operators discussed so far have $L^{p_{1}}\left(\mathbb{R}^{n}\right) \times L^{p_{2}}\left(\mathbb{R}^{n}\right), H^{1}\left(\mathbb{R}^{n}\right) \times$ $L^{p_{2}}\left(\mathbb{R}^{n}\right), L^{p_{1}}\left(\mathbb{R}^{n}\right) \times H^{1}\left(\mathbb{R}^{n}\right)$, or $H^{1}\left(\mathbb{R}^{n}\right) \times H^{1}\left(\mathbb{R}^{n}\right)$, with $1<$ $p_{1} \leq \infty$ and $1<p_{2} \leq \infty$, as domain of the operator. In analogy to the endpoint cases $p_{1}=1$ or $p_{2}=1$, natural spaces to consider as domain for indices below one are the Hardy spaces $H^{p}\left(\mathbb{R}^{n}\right)$, which are defined for $0<p \leq \infty$ in terms of maximal operators and coincide with $L^{p}\left(\mathbb{R}^{n}\right)$ for $1<p \leq \infty$ : given $\phi$ in the Schwartz class $\mathcal{S}\left(\mathbb{R}^{n}\right)$ such that $\int_{\mathbb{R}^{n}} \phi(x) d x \neq 0, f$ belongs to $H^{p}\left(\mathbb{R}^{n}\right)$ if

$$
\|f\|_{H^{p}}=\left\|\sup _{t>0}\left|\phi_{t} * f\right|\right\|_{L^{p}}<\infty,
$$

where $\phi_{t}(x)=t^{-n} \phi(x / t)$ for $x \in \mathbb{R}^{n}$ and $t>0$ and $\left(\phi_{t} * f\right)(x)=\int_{\mathbb{R}^{n}} \phi_{t}(x-y) f(y) d y$. The definition of $H^{p}\left(\mathbb{R}^{n}\right)$ is independent of the choice of $\phi$ and the corresponding norms are comparable.

What is the critical order for bilinear pseudodifferential operators with symbols in the Hörmander classes to be bounded from $H^{p_{1}}\left(\mathbb{R}^{n}\right) \times H^{p_{2}}\left(\mathbb{R}^{n}\right)$ into $L^{p}\left(\mathbb{R}^{n}\right)$ for $0<$ $p_{1} \leq \infty, 0<p_{2} \leq \infty$, and $0<p<\infty$ related through the Hölder condition (17)? Note that since the Hardy spaces coincide with the Lebesgue spaces for indices larger than one, the definition of the critical order must coincide with $m_{\rho}\left(p_{1}, p_{2}\right)$ for $p_{1}$ and $p_{2}$ larger than one. The answer was provided by A. Miyachi and N. Tomita [MT20, MT19] who proved that the extension of the critical order $m_{\rho}\left(p_{1}, p_{2}\right)$ to all positive values of $p_{1}$ and $p_{2}$ is given by

$$
m_{\rho}\left(p_{1}, p_{2}\right)=-n(1-\rho) \max \left\{\frac{1}{2}, \frac{1}{p_{1}}, \frac{1}{p_{2}}, \frac{1}{p^{\prime}}, \frac{1}{p}-\frac{1}{2}\right\},
$$

where $1 / p^{\prime}=1-1 / p$. See Figure 5 .

More precisely, the following result extends Theorem 2 and adds to the $L^{p}-H^{p}$ theory for bilinear pseudodifferential operators with symbols in the bilinear Hörmander classes.

Theorem 3. Let $0<p_{1} \leq \infty, 0<p_{2} \leq \infty$, and $0<p \leq \infty$ satisfy (17); consider $0 \leq \rho<1$. All bilinear pseudodifferential operators with symbols in $B S_{\rho, \rho}^{m}$ are bounded from $H^{p_{1}}\left(\mathbb{R}^{n}\right) \times$ $H^{p_{2}}\left(\mathbb{R}^{n}\right)$ into $L^{p}\left(\mathbb{R}^{n}\right)$ if and only if $m \leq m_{\rho}\left(p_{1}, p_{2}\right)$, where $L^{p}\left(\mathbb{R}^{n}\right)$ should be replaced by $B M O\left(\mathbb{R}^{n}\right)$ if $p=\infty$.

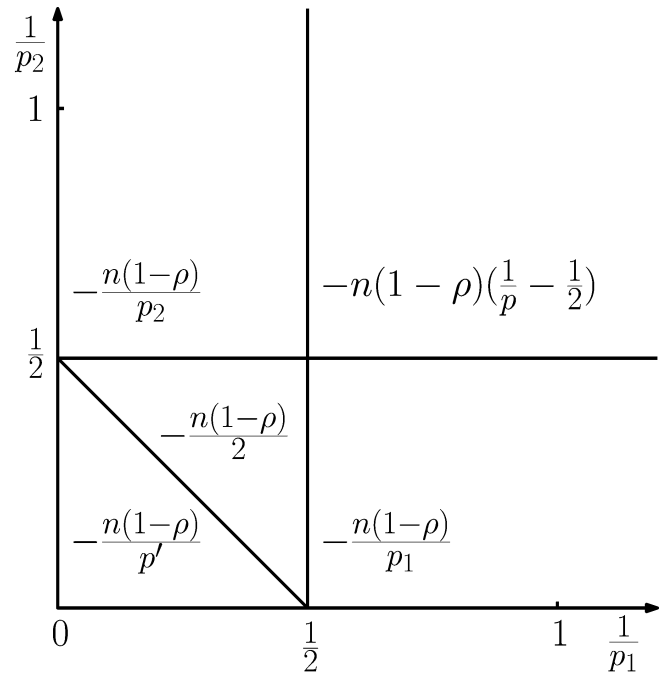

Figure 5. Visualization of $m_{\rho}\left(p_{1}, p_{2}\right)$ : for $\rho$ fixed, the diagram shows the values of $m_{\rho}\left(p_{1}, p_{2}\right)$ for $0<p_{1} \leq \infty, 0<p_{2} \leq \infty$, and $1 / p=1 / p_{1}+1 / p_{2}$.

Once more, in view of the symbolic calculus for the Hörmander classes, duality, and interpolation, along with the results of Theorem 2, the sufficiency of the condition $m \leq m_{\rho}\left(p_{1}, p_{2}\right)$ for boundedness follows from the cases corresponding to $0<p_{1}<1$ and $p_{2}=2$ with $m_{\rho}\left(p_{1}, 2\right)=-n(1-\rho) / p_{1}$, and $0<p_{1}<1$ and $p_{2}=\infty$ with $m_{\rho}\left(p_{1}, \infty\right)=-n(1-\rho) / p_{1}$. The reader is referred to A. Miyachi and N. Tomita [MT19] for a proof of these facts and to A. Miyachi and N. Tomita [MT20] for a proof of the necessity of the condition $m \leq m_{\rho}\left(p_{1}, p_{2}\right)$.

\section{Boundedness on Other Spaces}

As previously pointed out, operators with symbols in the class $B S_{1,1}^{0}$ are not necessarily bounded in the settings of Lebesgue spaces. However, boundedness results for such operators can be obtained in the context of function spaces involving smoothness conditions. For example, it was shown by Á. Bényi and R. H. Torres [BT03] that if $1<p_{1}<$ $\infty, 1<p_{2}<\infty$, and $1<p<\infty$ are related through the Hölder condition (17), $s>0$, and $\sigma \in B S_{1,1}^{0}$, it holds that

$$
\left\|T_{\sigma}(f, g)\right\|_{W^{s, p}} \lesssim\|f\|_{W^{s, p_{1}}}\|g\|_{L^{p_{2}}}+\|f\|_{L^{p_{1}}}\|g\|_{W^{s, p_{2}}} .
$$

Here $W^{s, p}\left(\mathbb{R}^{n}\right)$ is the Sobolev space defined as the class of functions $f$ in $L^{p}\left(\mathbb{R}^{n}\right)$ such that $J^{s} f \in L^{p}\left(\mathbb{R}^{n}\right)$, which coincides with the class of functions in $L^{p}\left(\mathbb{R}^{n}\right)$ that have distributional derivatives up to order $s$ in $L^{p}\left(\mathbb{R}^{n}\right)$ when $s \in \mathbb{N}$. In particular, (29) implies that $T_{\sigma}$ is bounded from $W^{s, p_{1}}\left(\mathbb{R}^{n}\right) \times W^{s, p_{2}}\left(\mathbb{R}^{n}\right)$ into $W^{s, p}\left(\mathbb{R}^{n}\right)$. Since $\|h\|_{W^{s, p}}=$ $\left\|J^{s} h\right\|_{L^{p}}$ for $h \in W^{s, p}\left(\mathbb{R}^{n}\right)$ and $T_{\tilde{\sigma}}(f, g)=f g$ for $\tilde{\sigma} \equiv 1$, the estimate (29) can be thought of as an extension of the fractional Leibniz rule (9) to all $T_{\sigma}$ with $\sigma \in B S_{1,1}^{0}$.

Estimates of the type (29) were proved by Á. Bényi for symbols in $B S_{1,1}^{0}$ in Besov and Lipschitz spaces, by the 
author [Nai15a] and by K. Koezuka and N. Tomita [KT18] for the class $B S_{1,1}^{m}$ in the scales of Triebel-Lizorkin and Besov spaces, and by A. Thomson and the author [NT19a] for symbols in the critical Hörmander classes in Besov spaces. The Triebel-Lizorkin spaces constitute a scale of spaces that contains the Sobolev, Lebesgue, and Hardy spaces as well as $B M O\left(\mathbb{R}^{n}\right)$ as particular cases while the scale of Besov spaces includes the Hölder spaces. All such estimates can be viewed in terms of Leibniz-type rules that extend (8) and (9).

Theorem 2 gives in particular that any operator with symbol in the critical class $B S_{0,0}^{-n / 2}$ is bounded from $L^{2}\left(\mathbb{R}^{n}\right) \times L^{2}\left(\mathbb{R}^{n}\right)$ into $L^{1}\left(\mathbb{R}^{n}\right)$. An improved result was given by A. Miyachi and N. Tomita [MT13] who showed that such operators are bounded from $L^{2}\left(\mathbb{R}^{n}\right) \times L^{2}\left(\mathbb{R}^{n}\right)$ into $h^{1}\left(\mathbb{R}^{n}\right)$, where $h^{1}\left(\mathbb{R}^{n}\right)$ is a local version of the Hardy space $H^{1}\left(\mathbb{R}^{n}\right)$ that is continuously contained in $L^{1}\left(\mathbb{R}^{n}\right)$. Other possible target spaces for boundedness in this case are $L^{p}\left(\mathbb{R}^{n}\right)$ with $1<p \leq 2$ (T. Kato, A. Miyachi, and N. Tomita) and Besov spaces (N. Hamada, N. Shida, and N. Tomita).

Finally, we note that pointwise inequalities of the type (27) imply boundedness properties of $T_{\sigma}$ in the context of weighted Lebesgue spaces leading to estimates such as

$$
\left\|T_{\sigma}(f, g)\right\|_{L_{w}^{p}} \lesssim\|f\|_{L_{w_{1}}^{p_{1}}}\|g\|_{L_{w_{2}}^{p_{2}}},
$$

where $\|h\|_{L_{u}^{q}}=\int_{\mathbb{R}^{n}}|h(x)|^{q} u(x) d x$ for a weight $u$, the indices $p_{1}, p_{2}$, and $p$ are related through the Hölder condition (17) with $2<p_{1}<\infty$ and $2<p_{2}<\infty, w_{1}$ and $w_{2}$ are appropriate weights in the so-called Muckenhoupt classes, and $w=w_{1}^{p / p_{1}} w_{2}^{p / p_{2}}$. Also, for operators with symbols in the Hörmander classes related to the bilinear Calderón-Zygmund theory, boundedness properties in weighted Lebesgue spaces associated to the Muckenhoupt classes and other classes of weights follow from such theory.

\section{Closing Remarks}

As we have seen, the bilinear Hörmander classes lead to a theory of bilinear pseudodifferential operators rich in techniques and applications. We also mention that there are a host of investigations on bilinear pseudodifferential operators with symbols satisfying diverse conditions and results leading to applications in analysis and partial differential equations.

Let us close with a list of open problems in connection with the bilinear Hörmander classes. As a first example, what are the minimum number of derivatives on the symbols for which the condition (10) should be required in order for the boundedness properties stated in Theorem 2 to hold true? Questions about minimal smoothness conditions in this sense have been partially studied by J. Herbert and the author as well as by T. Kato, A. Miyachi, and $\mathrm{N}$. Tomita for classes with $\rho=\delta=0$ and by T. Kato for
$B S_{\rho, \rho}^{-n(1-\rho) / 2}$. There is also a large amount of interest regarding minimal smoothness conditions for bilinear multipliers in terms of Sobolev norms and in the spirit of the linear Hörmander multiplier theorem as studied by M. Fujita, L. Grafakos, J. Hart, D. He, P. Honzík, A. Miyachi, H. V. Nguyen, B. Park, R. H. Torres, Z. Si, L. Slavíková, N. Tomita, and $\mathrm{X}$. Wu.

As another example, to the best of the author's knowledge, the corresponding theory for boundedness properties of operators with symbols in the bilinear Hörmander classes in the setting of weighted Lebesgue spaces has only been partially studied. As indicated above, some weighted estimates can be obtained from pointwise inequalities of the type (27) or from the bilinear Calderón-Zygmund theory; further weighted estimates follow from connections with bilinear fractional integral operators.

Other open questions are related to the symbolic calculus. As discussed, the bilinear Hörmander classes enjoy a symbolic calculus for transposes. On the other hand, the symbolic calculus for some natural compositions remains mostly open. More precisely, given $\mu, \nu, m \in \mathbb{R}$ and $0 \leq \delta \leq \rho \leq 1$, let $\sigma_{1}$ and $\sigma_{2}$ be in the linear Hörmander classes $S_{\rho, \delta}^{\mu}$ and $S_{\rho, \delta^{\prime}}^{\nu}$, respectively, assume $\sigma \in B S_{\rho, \delta}^{m}$, and consider bilinear operators such as

$$
\begin{aligned}
(f, g) & \mapsto T_{\sigma}\left(T_{\sigma_{1}}(f), T_{\sigma_{2}}(g)\right), \\
(f, g) & \mapsto T_{\sigma_{1}}\left(T_{\sigma}(f, g)\right) .
\end{aligned}
$$

The general question on how their corresponding symbols relate to the Hörmander classes or other classes of symbols remains open.

Expressions for the symbols of the compositions follow easily when some of the symbols in (30) and (31) are $x$-independent. For instance, the symbol of the operator in (30) is $\sigma(x, \xi, \eta) \sigma_{1}(\xi) \sigma_{2}(\eta)$ when $\sigma_{1}$ and $\sigma_{2}$ are $x$ independent; such symbol belongs to the class $B S_{\rho, \delta}^{\mu+\nu+m}$ when the support of $\sigma$ is contained in the set $\{(x, \xi, \eta)$ : $|\xi| \sim|\eta|\}$, to the class $B S_{\rho, \delta}^{\mu+m}$ when $\sigma_{2} \equiv 1$ and the support of $\sigma$ is contained in the set $\{(x, \xi, \eta):|\eta| \lesssim|\xi|\}$, and to the class $B S_{\rho, \delta}^{\nu+m}$ when $\sigma_{1} \equiv 1$ and the support of $\sigma$ is contained in the set $\{(x, \xi, \eta):|\xi| \lesssim|\eta|\}$. Similarly, the symbol of the operator in (31) when $\sigma_{1}$ and $\sigma$ are $x$-independent turns out to be $\sigma_{1}(\xi+\eta) \sigma(\xi, \eta)$ and such symbol is in $B S_{\rho, \delta}^{\mu+m}$ if $\sigma$ is supported in $\{(\xi, \eta):|\xi| \leq c|\eta|\}$ or $\{(\xi, \eta):|\eta| \leq c|\xi|\}$ for some $0<c<1$. However, without any assumptions, the symbols of the composition operators may fail to be in the "expected" classes of order $\mu+\nu+m, \mu+m$, or $v+m$. Indeed, as proved by Á. Benyi, A. Nahmod and R. H. Torres, if $\sigma_{1} \in S_{1,0}^{0}$ and $\sigma \in B S_{1,0}^{0}$, then the symbol in the composition (31) is not necessarily in $B S_{1,0}^{0}$ but rather in a class of symbols whose singularities are in the set where $\xi=\eta$; these classes of symbols are closely related to the bilinear Hilbert transform and were further studied 
by J. Gilbert and A. Nahmod [GN02] and C. Muscalu, T. Tao, and C. Thiele [MTT02].

As another example, bilinear operators with symbols satisfying conditions closely related to (4) model scattering properties of certain systems of PDEs associated to local and nonlocal operators of various different orders. Consider for instance the system

$$
\begin{cases}\partial_{t} u=v w, & u(0, x)=0 \\ \partial_{t} v+D^{s} v=0, & v(0, x)=f(x) \\ \partial_{t} w+D^{s} w=0, & w(0, x)=g(x)\end{cases}
$$

where $s>0$ and $f$ and $g$ are defined on $\mathbb{R}^{n}$. If $s$ is sufficiently large, then the solution $u$ scatters to a function $u_{\infty}$ that can be realized as $T_{\sigma_{s}}(f, g)$, where $\sigma_{s}$ is a CoifmanMeyer multiplier of order $-s$, that is,

$$
\left|\partial_{\xi}^{\beta} \partial_{\eta}^{\gamma} \sigma(\xi, \eta)\right| \leq C_{\beta, \gamma}(|\xi|+|\eta|)^{-s-(|\beta|+|\gamma|)}
$$

for every $\xi, \eta \in \mathbb{R}^{n}$ with $(\xi, \eta) \neq(0,0)$ and for $\beta, \gamma \in \mathbb{N}_{0}^{n}$ "large" enough.

As a consequence, boundedness properties of $T_{\sigma}$ for such $\sigma$ (as those studied by A. Thomson and the author [NT19b]) give rise to estimates of $u_{\infty}$ in terms of the data $f$ and $g$.

More generally, consider the system

$$
\begin{cases}\partial_{t} u+T_{a}(u)=v w, & u(0, x)=0 \\ \partial_{t} v+T_{b}(v)=0, & v(0, x)=f(x) \\ \partial_{t} w+T_{c}(w)=0, & w(0, x)=g(x)\end{cases}
$$

where $a, b$, and $c$ are multipliers defined in $\mathbb{R}^{n}$. Different choices of $a, b$, and $c$ under appropriate assumptions lead to $u_{\infty}$ being expressed as $T_{\sigma}(f, g)$, where $\sigma$ is a bilinear multiplier. For instance, choosing $a \equiv 0, b(\xi)=|\xi|^{s_{1}}$, and $c(\eta)=|\eta|^{s_{2}}$, where $s_{1}>0$ and $s_{2}>0, \sigma$ takes the form

$$
\sigma(\xi, \eta)=\left(|\xi|^{s_{1}}+|\eta|^{s_{2}}\right)^{-1}
$$

and it naturally leads to the study of a notion of anisotropic Coifman-Meyer multipliers.

ACKNOWLEDGMENTS. The author thanks Árpad Bényi, Fu Ken Ly, Diego Maldonado, Rodolfo Torres and the anonymous referees for their valuable comments and suggestions. The author was supported in part by the Simons Foundation grant 705953.

\section{References}

$\left[\mathrm{BBM}^{+} 13\right]$ Árpad Bényi, Frédéric Bernicot, Diego Maldonado, Virginia Naibo, and Rodolfo H. Torres, On the Hörmander classes of bilinear pseudodifferential operators II, Indiana Univ. Math. J. 62 (2013), no. 6, 1733-1764, DOI 10.1512/iumj.2013.62.5168. MR3205530
[BMNT10] Árpád Bényi, Diego Maldonado, Virginia Naibo, and Rodolfo H. Torres, On the Hörmander classes of bilinear pseudodifferential operators, Integral Equations Operator Theory 67 (2010), no. 3, 341-364, DOI $10.1007 / \mathrm{s} 00020-$ 010-1782-y. MR2660466

[BT03] Árpád Bényi and Rodolfo H. Torres, Symbolic calculus and the transposes of bilinear pseudodifferential operators, Comm. Partial Differential Equations 28 (2003), no. 5-6, 1161-1181, DOI 10.1081/PDE-120021190, MR1986065

[BT04] Árpád Bényi and Rodolfo H. Torres, Almost orthogonality and a class of bounded bilinear pseudodifferential operators, Math. Res. Lett. 11 (2004), no. 1, 1-11, DOI 10.4310/MRL.2004.v11.n1.a1. MR2046194

[CM78] Ronald R. Coifman and Yves Meyer, Au delà des opérateurs pseudo-différentiels (French), Astérisque, vol. 57, Société Mathématique de France, Paris, 1978. With an English summary. MR518170

[CV72] A. P. Calderón and R. Vaillancourt, A class of bounded pseudo-differential operators, Proc. Nat. Acad. Sci. U.S.A. 69 (1972), 1185-1187. MR298480

[Fef73] Charles Fefferman, $L^{p}$ bounds for pseudo-differential operators, Israel J. Math. 14 (1973), 413-417, DOI 10.1007/BF02764718. MR336453

[GN02] John E. Gilbert and Andrea R. Nahmod, $L^{p}$ boundedness for time-frequency paraproducts. II, J. Fourier Anal. Appl. 8 (2002), no. 2, 109-172, DOI 10.1007/s00041-002-0006-5. MR1891727

[GO14] L. Grafakos and S. Oh, The Kato-Ponce inequality, Comm. Partial Differential Equations 39 (2014), no. 6, 1128-1157. MR3200091

[GT02] L. Grafakos and R. H. Torres, Multilinear CalderónZygmund theory, Adv. Math. 165 (2002), no. 1, 124-164. MR1880324 (2002j:42029)

[Hör67] L. Hörmander, Pseudo-differential operators and hypoelliptic equations, Singular integrals, Proc. Sympos. Pure Math., Vol. X, (Chicago, Ill., 1966), American Mathematical Society, Providence, RI, 1967, pp. 138-183. MR0383152

[KP88] T. Kato and G. Ponce, Commutator estimates and the Euler and Navier-Stokes equations, Comm. Pure Appl. Math. 41 (1988), no. 7, 891-907. MR951744 (90f:35162)

[KT18] K. Koezuka and N. Tomita, Bilinear pseudo-differential operators with symbols in $B S_{1,1}^{m}$ on Triebel-Lizorkin spaces, J. Fourier Anal. Appl. 24 (2018), no. 1, 309-319. MR3750316

[MRS14] N. Michalowski, D. Rule, and W. Staubach, Multilinear pseudodifferential operators beyond Calderón-Zygmund theory, J. Math. Anal. Appl. 414 (2014), no. 1, 149-165. MR3165300

[MT13] A. Miyachi and N. Tomita, Calderón-Vaillancourt-type theorem for bilinear operators, Indiana Univ. Math. J. 62 (2013), no. 4, 1165-1201. MR3179688

[MT20] Akihiko Miyachi and Naohito Tomita, Bilinear pseudo-differential operators with exotic symbols, Ann. Inst. Fourier (Grenoble) 70 (2020), no. 6, 2737-2769. MR4245629 


\section{NOW AVAILABLE FROM HINDUSTAN
HOOK AGENCY}

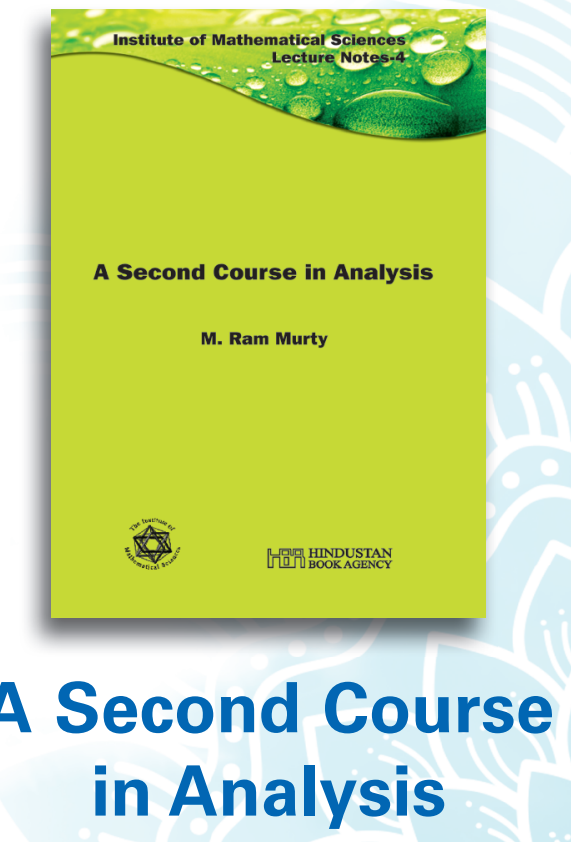

\section{Ram Murty, Queen's University, Kingston, ON, Canada}

This book contains material suitable for a two-semester graduate course in analysis. Based on courses given by the author since 2007, it is targeted towards graduate students preparing for a research career in mathematics.

Hindustan Book Agency; 2020; 350 pages: Softcover; ISBN: 978-93-86279-84-2; List US\$68; AMS members US\$54.40; Order code HIN/81

Titles published by the Hindustan Book Agency (New Delhi, India) include studies in advanced mathematics, monographs, lecture notes, and/or conference proceedings on current topics of interest.

\section{Discover more books at bookstore.ams.org/hin}

Publications of Hindustan Book Agency are distributed within the Americas by the American Mathematical Society. Maximum discount of $20 \%$ for commercial channels.

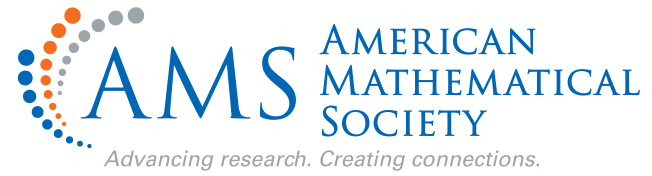

[MT19] Akihiko Miyachi and Naohito Tomita, Bilinear pseudo-differential operators with exotic symbols, II, J. PseudoDiffer. Oper. Appl. 10 (2019), no. 2, 397-413, DOI 10.1007/s11868-018-0251-2. MR3945928

[MTT02] Camil Muscalu, Terence Tao, and Christoph Thiele, Multi-linear operators given by singular multipliers, J. Amer. Math. Soc. 15 (2002), no. 2, 469-496, DOI 10.1090/S0894-0347-01-00379-4. MR1887641

[Nai15a] Virginia Naibo, On the bilinear Hörmander classes in the scales of Triebel-Lizorkin and Besov spaces, J. Fourier Anal. Appl. 21 (2015), no. 5, 1077-1104, DOI 10.1007/s00041015-9398-x. MR3393696

[Nai15b] Virginia Naibo, On the $L^{\infty} \times L^{\infty} \rightarrow B M O$ mapping property for certain bilinear pseudodifferential operators, Proc. Amer. Math. Soc. 143 (2015), no. 12, 5323-5336, DOI 10.1090/proc12775. MR3411149

[NT19a] Virginia Naibo and Alexander Thomson, Bilinear Hörmander classes of critical order and Leibniz-type rules in Besov and local Hardy spaces, J. Math. Anal. Appl. 473 (2019), no. 2, 980-1001, DOI 10.1016/j.jmaa.2019.01.005. MR3912862

[NT19b] Virginia Naibo and Alexander Thomson, CoifmanMeyer multipliers: Leibniz-type rules and applications to scattering of solutions to PDEs, Trans. Amer. Math. Soc. 372 (2019), no. 8, 5453-5481, DOI 10.1090/tran/7866 MR4014283

[Tor20] Rodolfo H. Torres, Almost-orthogonality in Fourier analysis: from discrete characterizations of function spaces, to singular integrals, to Leibniz rules for fractional derivatives, Notices Amer. Math. Soc. 67 (2020), no. 8, 1105-1115, DOI 10.1090/noti MR4187859

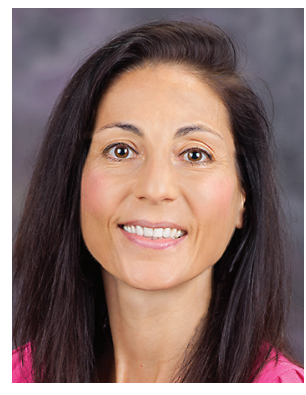

Virginia Naibo

Credits

Opening image is courtesy of ermess via Getty.

Figures 1-5 are courtesy of Virginia Naibo.

Photo of Virginia Naibo is courtesy of Kansas State University, Communications and Marketing. 